\title{
Acute imaging does not improve ASTRAL score's accuracy despite having a prognostic value
}

\author{
George Ntaios ${ }^{1 *}{ }^{\dagger}$, Vasileios Papavasileiou ${ }^{1+}$, Mohamed Faouzi $^{2}$, Peter Vanacker ${ }^{3}$, \\ Max Wintermark ${ }^{4}$, and Patrik Michel ${ }^{5}$
}

\begin{abstract}
Background The ASTRAL score was recently shown to reliably predict three-month functional outcome in patients with acute ischemic stroke.

Aim The study aims to investigate whether information from multimodal imaging increases ASTRAL score's accuracy.

Methods All patients registered in the ASTRAL registry until March 2011 were included. In multivariate logistic-regression analyses, we added covariates derived from parenchymal, vascular, and perfusion imaging to the 6-parameter model of the ASTRAL score. If a specific imaging covariate remained an independent predictor of three-month modified Rankin score $>2$, the area-under-the-curve (AUC) of this new model was calculated and compared with ASTRAL score's AUC. We also performed similar logistic regression analyses in arbitrarily chosen patient subgroups.

Results When added to the ASTRAL score, the following covariates on admission computed tomography/magnetic resonance imaging-based multimodal imaging were not significant predictors of outcome: any stroke-related acute lesion, any nonstroke-related lesions, chronic/subacute stroke,
\end{abstract}

Correspondence: George Ntaios*, Department of Medicine, University of Thessaly, Biopolis 41110, Larissa, Greece.

E-mail: gntaios@med.uth.gr

${ }^{1}$ Department of Medicine, University of Thessaly, Larissa, Greece

${ }^{2}$ Institute of Social and Preventive Medicine, Centre Hospitalier Universitaire Vaudois and University of Lausanne, Lausanne, Switzerland

${ }^{3}$ Department of Neurology, University Hospital Antwerp, Edegem, Belgium

${ }^{4}$ Department of Radiology, Division of Neuroradiology, University of Virginia, Charlottesville, VA, USA

${ }^{5}$ Stroke Center, Neurology Service, Department of Neurosciences, Centre Hospitalier Universitaire Vaudois and University of Lausanne, Lausanne, Switzerland

Received: 30 January 2014; Accepted: 29 April 2014; Published online 3 June 2014

${ }^{\dagger}$ G. Ntaios and V. Papavasileiou have contributed equally and are co-first authors.

Funding: Dr P. Michel has received through his employer (CHUV) research grants from the Swiss National Science Foundation, the Swiss Heart Foundation, and Cardiomet-CHUV for analyses based on the ASTRAL registry. The other co-authors report no disclosure.

Authors' contributions: G. Ntaios: Study concept and design, analysis and interpretation, preparation of the manuscript, study supervision. V. Papavasileiou: Data acquisition, analysis and interpretation, critical revision of the manuscript for important intellectual content. M. Faouzi: Analysis and interpretation, critical revision of the manuscript for important intellectual content. P. Vanacker: Data acquisition, critical revision of the manuscript for important intellectual content. M. Wintermark: Study concept and design, critical revision of the manuscript for important intellectual content. P. Michel: Study concept and design, data acquisition, analysis and interpretation, critical revision of the manuscript for important intellectual content, study supervision.

DOI: 10.1111/ijs.12304 leukoaraiosis, significant arterial pathology in ischemic territory on computed tomography angiography/magnetic resonance angiography/Doppler, significant intracranial arterial pathology in ischemic territory, and focal hypoperfusion on perfusion-computed tomography. The Alberta Stroke Program Early CT score on plain imaging and any significant extracranial arterial pathology on computed tomography angiography/magnetic resonance angiography/Doppler were independent predictors of outcome (odds ratio: $0.93,95 \% \mathrm{Cl}$ : 0.87-0.99 and odds ratio: $1.49,95 \% \mathrm{Cl}: \mathbf{1 . 0 8}-2 \cdot 05$, respectively) but did not increase ASTRAL score's AUC ( 0.849 vs. 0.850 , and 0.8563 vs. $\mathbf{0 . 8 5 6 4}$, respectively). In exploratory analyses in subgroups of different prognosis, age or stroke severity, no covariate was found to increase ASTRAL score's AUC, either.

Conclusions The addition of information derived from multimodal imaging does not increase ASTRAL score's accuracy to predict functional outcome despite having an independent prognostic value. More selected radiological parameters applied in specific subgroups of stroke patients may add prognostic value of multimodal imaging.

Key words: ASTRAL score, CT angiography, CT, functional outcome, multimodal imaging, perfusion CT

\section{Introduction}

Multimodal imaging (MMI) of acute ischemic stroke (AIS) including noninvasive arterial and perfusion imaging shows promise to improve stroke diagnosis, prognosis, and treatment selection of AIS patients (1-3). With regard to prognosis, the value of the presence, site and extent of arterial occlusion (4-6), of the infarct core (7-9), and of penumbra (8-10) have been shown to be useful for prediction of stroke outcome in selected patient populations.

The ASTRAL score was introduced recently for the prognosis of functional outcome in patients with AIS (11). It is based on six nonradiological items readily available upon arrival of the stroke patient in the emergency department (11). It has been externally validated in three independent and ethnically diverse cohorts, showing remarkable consistency on predicting three-month functional outcome $(11,12)$; recently, it was also validated externally for the prognosis of five-year dependence and mortality (13). Other scores have also been introduced to predict ischemic stroke outcome like the PLAN (14), the iSCORE (15), the SSV (16), the MOSAIC (17), and others (18-22). Most, but not all (17), of these current stroke outcome scores omit imaging technology to remain simple and rapid (11). This is especially the case for the ASTRAL score which can be easily calculated at bedside without any mathematical calculation by using the color chart which may be found in the original publication of the score (11).

The aim of the present study was to investigate whether the addition of information from multimodal imaging increases 
ASTRAL score's prognostic accuracy to predict three-month functional outcome in patients with acute ischemic stroke.

\section{Patients and methods}

\section{Data selection}

All patients who were admitted with AIS between January 2003 and March 2011 and had a prestroke modified Rankin Scale score $(\mathrm{mRS}) \leq 2$ were selected from the Acute STroke Registry and Analysis of Lausanne (ASTRAL). ASTRAL is the prospective registry of all AIS patients admitted to the stroke unit and/or intensive care unit of the Lausanne University Hospital (CHUV) within $24 \mathrm{~h}$ after last proof of well-being (23). Patients with missing three-month mRS and one or more missing component(s) needed to calculate the ASTRAL score were excluded from the analysis. The scientific use of the data registered in ASTRAL was approved by the ethics commission (subcommision III) for research on humans of the Canton of Vaud.

As previously described (23), the ASTRAL score was calculated based on age (1 point for every five-years), stroke severity [ 1 point for every point of National Institutes of Health Stroke Scale score (NIHSS) at admission], time delay between symptom onset and admission ( 2 points if $>$ three-hours), presence of any new visual field defect ( 2 points), glucose at admission ( 1 point if $>7.3 \mathrm{mmol} / \mathrm{l}$ or $<3.7 \mathrm{mmol} / \mathrm{l}$ ), and level of consciousness (3 points if impaired item 1a on the NIHSS was $>0$ ) (11).

The methods applied to collect and register data, the definitions used in the ASTRAL registry, and the imaging protocols for acute ischemic stroke in our center were previously described (23). With regard to acute MMI, for all patients, parenchymal imaging [mostly computed tomography (CT)] was analyzed if performed within $24 \mathrm{~h}$ after stroke onset. The presence of early ischemic changes, the Alberta Stroke Program Early CT score (ASPECTS) (24), the presence of chronicl subacute stroke and of leukoaraiosis [Blenow grade 1 or more (25)] were registered. Acute vascular imaging [mostly CT angiography (CTA)] within the first $24 \mathrm{~h}$ after stroke onset was analyzed and classified in significant arterial pathology in extraand intracranial arteries. Arterial pathology was considered significant if occlusion, $>50 \%$ stenosis, or any signs of dissection were present; these parameters were combined into a single category to simplify their use in clinical practice and increase the statistical power of the study. Also, perfusion CT (PCT) was performed in most patients with symptoms not clinically limited to the posterior fossa, according to methods described elsewhere $(26,27)$. The presence of focal hypoperfusion on visually inspected slices of cerebral blood flow measurement (corresponding to the combination of infarct core and penumbra) was noted as present or absent. Threshold maps for core and penumbra were not calculated for this project. All patients were treated according to current European Stroke Organization guidelines (28). Unfavorable functional outcome was defined as three-month $\mathrm{mRS}>2$.

\section{Statistical analysis}

To investigate the prognostic accuracy of the ASTRAL score to predict three-month functional outcome, we assessed the dis- criminatory power of the score, i.e., the degree to which the prognostic score enables the discrimination between patients with favorable and unfavorable outcome by calculating the areaunder-the-curve (AUC).

To assess if a specific imaging covariate increases the prognostic accuracy of the ASTRAL score, we first performed a multivariate logistic regression analysis with the specific imaging covariate added in the 6-parameter model of the ASTRAL score (i.e., age, NIHSS score, onset-to-admission time interval, visual field defect, glucose and level of consciousness). In case that a specific imaging covariate remained an independent predictor of three-month functional outcome, the AUC of this new model (i.e., the ASTRAL score plus the specific imaging covariate) was calculated and compared with the AUC of the 6-parameter model of the ASTRAL score. A specific covariate would be considered that it increases the prognostic accuracy of the ASTRAL score if the AUC of the new model was significantly higher than the AUC of the ASTRAL score. The statistical comparison of the AUCs was performed with the DeLong method (29). The imaging covariates included in the multivariate logistic regression analyses were the following: any stroke-related acute lesion on CT or magnetic resonance imaging (MRI), the ASPECTS, any vascular lesions on acute CT or MRI not related to the current stroke (i.e., chronic or subacute stroke or leukoaraiosis), chronic or subacute stroke on acute CT or MRI, leukoaraiosis, focal hypoperfusion on PCT, significant arterial pathology in the ischemic territory in acute CTA or magnetic resonance angiography (MRA) or Doppler, significant intracranial arterial pathology in the ischemic territory in acute CTA or MRA or Doppler, and significant extracranial arterial pathology in the ischemic territory in acute CTA or MRA or Doppler. The ASPECTS was analyzed both as continuous and categorical covariate using different arbitrary cutoffs to reduce the possibility of a type II error (false negative result).

We also investigated whether imaging increased the prognostic accuracy of the ASTRAL score in specific patient subgroups. Hence we performed similar logistic regression analyses in arbitrarily chosen subgroups of patients with regard to their age $(<71$ and $\geq 71$ years), NIHSS at admission $(\leq 3,4-19$, and $>19)$, and ASTRAL score (0-22, 23-38, and >38).

For continuous variables, data are summarized as medians and interquartile range (IQR). Categorical data are presented as absolute numbers and percentage. Imputation techniques were not implemented for missing data. In the multivariate analyses, the level of statistical significance was set at 95\% $(P=0 \cdot 05)$. Associations are presented as odds ratios (OR) with $95 \%$ confidence intervals (95\% CI). The statistical analysis was performed with STATA Statistical Software (Release 10, College Station, TX, USA: StataCorp LP 2007).

\section{Results}

From the entire cohort of 2401 patients in the ASTRAL registry during the observation period, 203 patients were excluded because of a prestroke mRS $>2$ and 95 patients because the initial imaging was performed $>24 \mathrm{~h}$ after stroke onset. One hundred 
sixty-four patients were excluded because one or more of the six components of the ASTRAL score were unavailable and 47 because three-month mRS was not available. Finally, 1892 patients [median age: $71 \cdot 2$ years, IQR: $59 \cdot 4-79 \cdot 8$; 1094 (57.8\%) males] were available for analysis. Overall, 1855 (98.0\%) had an acute CT, 1626 (85.9\%) had acute CTA, and 1268 (67.1\%) had PCT. A few patients had MRI as the initial imaging, and others had a CT followed by MRI in the acute phase. Three hundred eighty-one $(20 \cdot 1 \%)$ patients were treated with intravenous thrombolysis and $31(1 \cdot 6 \%)$ with endovascular revascularization. The baseline characteristics of the patients are summarized in Table 1.

The AUC of the ASTRAL score in the overall population was $0 \cdot 850$, which is similar with the AUC of the score during the analysis which introduced the ASTRAL score (11). When added to the ASTRAL score in logistic regression analyses, the following covariates were not significant predictors of three-month functional outcome: any stroke-related acute lesion on CT or MRI, any vascular lesions on acute CT or MRI not related to the current stroke, significant arterial pathology in the ischemic territory, significant intracranial arterial pathology in the ischemic territory, and focal hypoperfusion on PCT. There was no interaction between any of the aforementioned covariates and time delay since stroke onset.

In a logistic regression analysis including the ASTRAL score as a covariate, significant extracranial arterial pathology in the ischemic territory on acute CTA or MRA or Doppler was an independent predictor of three-month functional outcome (OR: 1.49, 95\% CI: $1 \cdot 08-2 \cdot 05, P=0 \cdot 014)$. However, the AUC of the combined model (which included both covariates) was similar to the AUC of the ASTRAL score $(0 \cdot 849$ vs. $0 \cdot 850)$, which means that significant extracranial arterial pathology does not increase significantly the prognostic accuracy of the ASTRAL score (despite being an independent predictor).

There were 1262 patients with an ASPECTS of 10, and 215 patients with an ASPECTS of $<7$. In a logistic regression analysis including the ASTRAL score as a covariate, the ASPECTS - when analyzed as a continuous covariate - was a significant predictor of three-month functional outcome (OR: 0.93, 95\% CI: 0.87-0.99, $P=0.023$ ). However, the AUC of the combined model (which included both covariates) was similar to the AUC of the ASTRAL score $(0.8563$ vs. $0.8564, P=0.893)$, which means that the ASPECTS (as a continuous covariate) does not increase significantly the prognostic accuracy of the ASTRAL score (despite being an independent predictor).

The ASPECTS was also analyzed as a categorical covariate using different cutoff values $(3,5,7$, and 9) in a logistic regression analysis including the ASTRAL score as a covariate. For the cutoffs 3 and 5, it was a significant predictor of three-month functional outcome (OR: $2 \cdot 32,95 \%$ CI: $1 \cdot 15-4 \cdot 65, P=0.018$ and OR: 1.95 , 95\% CI: $1 \cdot 21-3 \cdot 12, P=0 \cdot 006$, respectively). However, the AUC of the combined model (which included both covariates) was similar to the AUC of the ASTRAL score $(0 \cdot 857$ vs. 0.856 , $P=0 \cdot 318$, for the cutoff value of $3 ; 0 \cdot 857$ vs. $0 \cdot 856, P=0 \cdot 5695$, for the cutoff value of 5). For the cutoffs of 7 and 9, the ASPECTS was not a significant predictor of three-month outcome.
Table 1 Baseline characteristics of analyzed patients

\begin{tabular}{|c|c|}
\hline Total number of eligible patients & 1892 \\
\hline Median age (years) & $71 \cdot 2(59 \cdot 4-79 \cdot 8)$ \\
\hline Male gender & $1094(57 \cdot 8 \%)$ \\
\hline NIHSS at admission & $6(3-14)$ \\
\hline Onset-to-admission time (minutes) & $204(93-624)$ \\
\hline \multicolumn{2}{|l|}{ Prestroke mRS } \\
\hline 0 & $1215(64 \cdot 3 \%)$ \\
\hline 1 & $472(24.9 \%)$ \\
\hline 2 & $205(10 \cdot 8 \%)$ \\
\hline Serum glucose at admission (mmol/l) & $6 \cdot 5(5 \cdot 7-7 \cdot 7)$ \\
\hline \multicolumn{2}{|l|}{ Blood pressure at admission ( $\mathrm{mmHg}$ ) } \\
\hline Systolic & $157(140-176)$ \\
\hline Diastolic & $89(78-100)$ \\
\hline Visual field deficit & $635(33 \cdot 6 \%)$ \\
\hline Decreased level of consciousness & $200(10 \cdot 6 \%)$ \\
\hline \multicolumn{2}{|l|}{ Stroke risk factors } \\
\hline Smoking* & $460(24 \cdot 5 \%)$ \\
\hline Hypertension $^{\dagger}$ & $1266(67 \cdot 1 \%)$ \\
\hline Atrial fibrillation $(\mathrm{AF})^{\ddagger}$ & $478(25 \cdot 3 \%)$ \\
\hline Diabetes mellitus & $312(16 \cdot 6 \%)$ \\
\hline Dyslipidemia & $1299(68.8 \%)$ \\
\hline Acute $C T<24$ h of onset & $1855(98.0 \%)$ \\
\hline \multicolumn{2}{|l|}{ Acute arterial imaging performed } \\
\hline Any vascular imaging & $1695(89 \cdot 7 \%)$ \\
\hline None & $195(10 \cdot 3 \%)$ \\
\hline CTA & $1626(85 \cdot 9 \%)$ \\
\hline MRA & $66(3 \cdot 5 \%)$ \\
\hline Doppler & $333(17 \cdot 6 \%)$ \\
\hline Perfusion CT performed & $1268(67 \cdot 1 \%)$ \\
\hline \multicolumn{2}{|l|}{ ASPECTS score on admission } \\
\hline 10 & $1262(67 \cdot 8 \%)$ \\
\hline $7-9$ & $383(20 \cdot 6 \%)$ \\
\hline$<7$ & $215(11 \cdot 6 \%)$ \\
\hline \multicolumn{2}{|l|}{ Stroke mechanism (TOAST classification) } \\
\hline Large-artery atherosclerotic stroke & $265(14 \cdot 1 \%)$ \\
\hline Cardioembolic stroke & $624(33 \cdot 1 \%)$ \\
\hline Lacunar stroke & $263(14.0 \%)$ \\
\hline Stroke of other determined etiology & $72(3 \cdot 8 \%)$ \\
\hline $\begin{array}{l}\text { Stroke of undetermined origin or multiple } \\
\text { etiologies }\end{array}$ & $659(35.0 \%)$ \\
\hline \multicolumn{2}{|l|}{ Treatment } \\
\hline Intravenous thrombolysis & $381(20 \cdot 1 \%)$ \\
\hline Endovascular revascularization & $31(1.6 \%)$ \\
\hline
\end{tabular}

*Defined as current smoking or smoking stopped $<2$ years before the stroke.

${ }^{\dagger}$ Known or newly diagnosed hypertension.

${ }^{\ddagger}$ Known history or newly diagnosed AF; patients with one single episode of AF are not included.

Numbers represent medians and interquartile range for continuous covariates, and absolute count and proportion for categorical covariates

ASPECTS, Alberta Stroke Program Early CT score; CT, computed tomography; CTA, CT angiography; MRA, magnetic resonance angiography; mRS, modified Rankin Scale score; NIHSS, National Institute of Health Stroke Scale score.

In exploratory logistic regression analyses, to identify arbitrarily chosen subgroups of patients in which the addition of imaging could increase the prognostic accuracy of the ASTRAL score, no covariate was found to significantly increase the prognostic accuracy of the ASTRAL score in any subgroup (Table 2). 


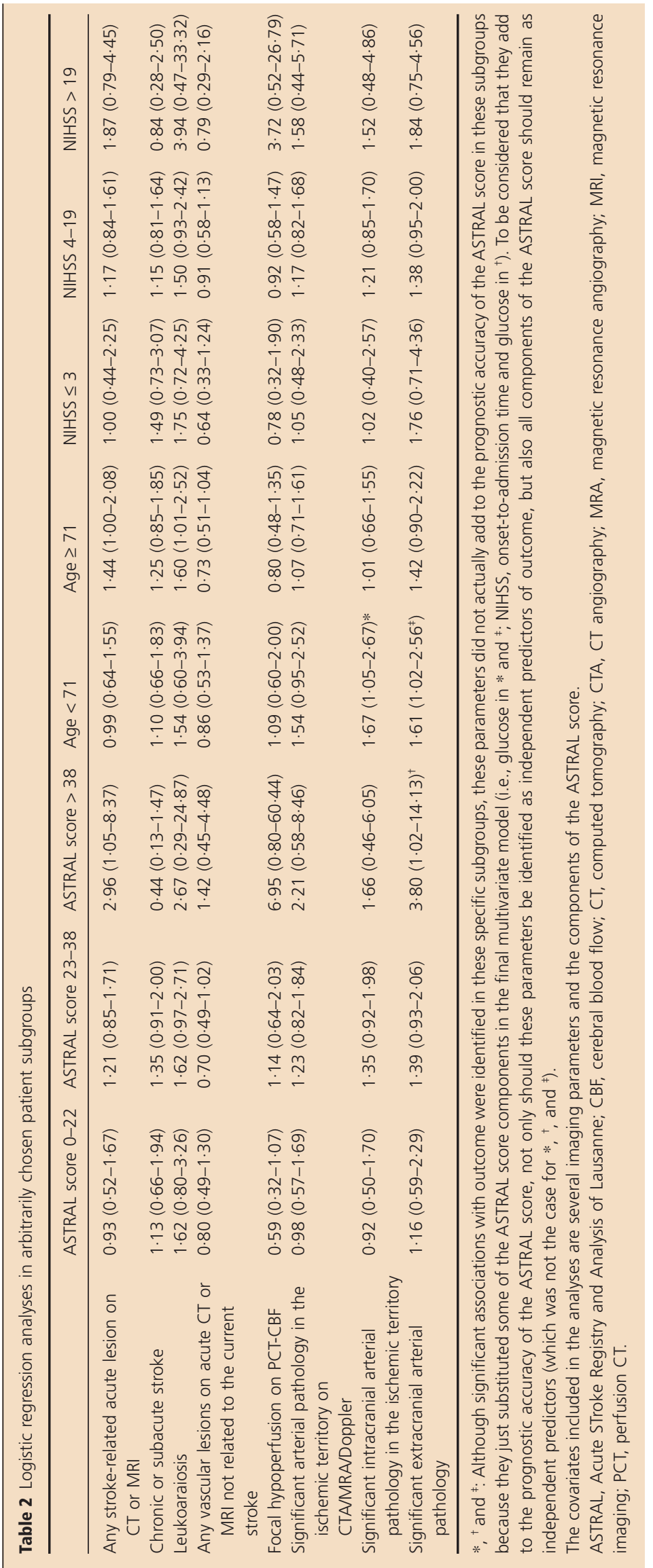




\section{Discussion}

The present study analyzed a large series of previously independent consecutive AIS patients and found that the addition of readily available information derived from mostly CT-based MMI does not increase the accuracy of the ASTRAL score to predict three-month functional outcome despite the fact that certain imaging parameters (i.e., the ASPECTS and the presence of extracranial arterial pathology in the ischemic territory) had an independent prognostic value.

The fact that the prognostic accuracy of the ASTRAL score did not increase by the addition of information from (parenchymal, vascular, and perfusion) MMI neither in the overall study population nor in selected arbitrarily chosen patient subgroups, should only be regarded as a further confirmation of the excellent performance of the ASTRAL score, and, of course, by no means does it suggest that acute imaging does not need to be performed during acute stroke. The most plausible explanation for this finding could be that most of the information derived from acute MMI is already incorporated into (certain components of) the ASTRAL score: there is a causal chain in acute ischemic stroke which originates from the vascular lesion and predicts clinical severity and imaging findings. The ASTRAL score gives considerable weight to the initial NIHSS and level of consciousness which were previously shown to be closely associated with the presence of acute intracranial arterial occlusions (30) and with the extent of core and penumbra $(10,31,32)$. Therefore, the clinical variables included in the ASTRAL score may eliminate MMI radiological covariates from being independent predictors, or make them of little additive value. It may also be that we did not analyze sufficient details of MMI, such as threshold-based volumes of nonviable and at-risk tissue, site and extent of occlusion, or collaterals. Also, it may be due to the fact that we did not target specific stroke types but have analyzed a consecutive real-world AIS population. Still, the analysis of defined subgroups within our population did not improve the prognostic score either.

We found that the ASPECTS based on plain imaging and the presence of significant extracranial arterial pathology on CTA/ MRA/Doppler were independent predictors of functional outcome. These results confirm previous observations that the ASPECTS [based on noncontrast CT (24)] and diffusion weighted imaging (DWI) (33) are prognostic predictors independently of clinical and epidemiological variables. Regarding other parameters of MMI, we limited our analysis to readily available parameters to avoid the additional time required for detailed analysis and the sometimes difficult decisions of thresholds (34). Doing so, the presence of any extracranial stenosis $\geq 50 \%$ or occlusion was associated with worse three-month prognosis, but intracranial arterial pathology or focal tissue hypoperfusion was not. Other MMI parameters did not add independent information in this consecutive AIS population. MMI in AIS has been shown in some studies to add prognostic value in addition to clinical parameters $(8,10,35)$. Collateral status, which we did not measure, was also an independent predictor in some studies $(36,37)$ as were certain threshold tissue viability parameters $(10)$. These MMI parameters which will require more time and exper- tise to assess could be added in further similar studies. Moreover, MMI was shown to predict response to revascularization treatment in selected stroke patients and situations, such as mismatch imaging in territorial infarct of the middle cerebral artery (MCA) territory with visible hypoperfusion (38-41) or arterial imaging in MCA strokes before thrombolysis (6); our study was not designed to address the question of treatment response.

Strengths of the present study are the inclusion and analysis of a large series of consecutive AIS over an eight-year period. Also, we analyzed information derived from MMI (including vascular and perfusion imaging) performed during the acute phase of stroke (within $24 \mathrm{~h}$ after stroke onset). Moreover, this analysis was performed on the same dataset which was used to develop the ASTRAL score (11). Also, we tried several statistical analyses to identify potential covariates which could increase the prognostic accuracy of the ASTRAL score, e.g., the ASPECTS was analyzed both as a continuous and a categorical covariate using different cutoffs. Finally, we also analyzed several patient subgroups to identify potential populations for which the addition of imaging could increase the predictive accuracy of the ASTRAL score.

Limitations of the study are that information about the volume of the initial infarct and the penumbra, the degree of vascular stenosis, thrombus length in occlusions, and a clot burden score were not available for the analysis. Also, information about collateral circulation in patients with intracranial occlusions, which was previously shown to predict stroke outcome $(36,42)$, was not available for the analysis. Therefore, it cannot be excluded that more specific imaging may eventually increase the prognostic accuracy of the ASTRAL score. Moreover, not all patients had available all imaging examinations (i.e., parenchymal, vascular, and perfusion brain imaging), which, however, would be impossible to achieve given the large series of included patients and the pragmatic real-world nature of this study. Finally, the present analysis was performed in the same patient population which was used to develop the ASTRAL score, which may have introduced selection bias.

In conclusion, the ASTRAL score is a reliable tool to predict three-month functional outcome in patients with AIS; further studies are necessary to investigate whether collateral or penumbral imaging could increase its prognostic accuracy.

\section{References}

1 Wintermark M, Albers GW, Broderick JP et al. Acute stroke imaging research roadmap II. Stroke 2013; 44:2628-39.

2 Albers GW, Thijs VN, Wechsler L et al. Magnetic resonance imaging profiles predict clinical response to early reperfusion: the diffusion and perfusion imaging evaluation for understanding stroke evolution (DEFUSE) study. Ann Neurol 2006; 60:508-17.

3 De Silva DA, Fink JN, Christensen S et al. Assessing reperfusion and recanalization as markers of clinical outcomes after intravenous thrombolysis in the echoplanar imaging thrombolytic evaluation trial (EPITHET). Stroke 2009; 40:2872-4.

4 Puetz V, Dzialowski I, Hill MD et al. Intracranial thrombus extent predicts clinical outcome, final infarct size and hemorrhagic transformation in ischemic stroke: the clot burden score. Int J Stroke 2008; 3:230-6.

5 Riedel CH, Zimmermann P, Jensen-Kondering U, Stingele R, Deuschl G, Jansen $O$. The importance of size: successful recanalization by 
intravenous thrombolysis in acute anterior stroke depends on thrombus length. Stroke 2011; 42:1775-7.

6 Fiebach JB, Al-Rawi Y, Wintermark M et al. Vascular occlusion enables selecting acute ischemic stroke patients for treatment with desmoteplase. Stroke 2012; 43:1561-6.

7 Jovin TG, Yonas H, Gebel JM et al. The cortical ischemic core and not the consistently present penumbra is a determinant of clinical outcome in acute middle cerebral artery occlusion. Stroke 2003; 34:2426-33.

8 Yoo AJ, Barak ER, Copen WA et al. Combining acute diffusionweighted imaging and mean transmit time lesion volumes with National Institutes of Health Stroke Scale Score improves the prediction of acute stroke outcome. Stroke 2010; 41:1728-35.

9 Mlynash M, Lansberg MG, De Silva DA et al. Refining the definition of the malignant profile: insights from the DEFUSE-EPITHET pooled data set. Stroke 2011; 42:1270-5.

10 Zhu G, Michel P, Aghaebrahim A et al. Computed tomography workup of patients suspected of acute ischemic stroke: perfusion computed tomography adds value compared with clinical evaluation, noncontrast computed tomography, and computed tomography angiogram in terms of predicting outcome. Stroke 2013; 44:104955 .

11 Ntaios G, Faouzi M, Ferrari J, Lang W, Vemmos K, Michel P. An integer-based score to predict functional outcome in acute ischemic stroke: the ASTRAL score. Neurology 2012; 78:1916-22.

12 Liu G, Ntaios G, Zheng $\mathrm{H}$ et al. External validation of the ASTRAL score to predict 3- and 12-month functional outcome in the China National Stroke Registry. Stroke 2013; 44:1443-5.

13 Papavasileiou V, Milionis H, Michel P et al. ASTRAL score predicts 5 -year dependence and mortality in acute ischemic stroke. Stroke 2013; 44:1616-20.

14 O'Donnell MJ, Fang J, D'Uva C et al. The PLAN score: a bedside prediction rule for death and severe disability following acute ischemic stroke. Arch Intern Med 2012; 172:1548-56.

15 Saposnik G, Raptis S, Kapral MK et al. The iScore predicts poor functional outcomes early after hospitalization for an acute ischemic stroke. Stroke 2011; 42:3421-8.

16 Counsell C, Dennis M, McDowall M, Warlow C. Predicting outcome after acute and subacute stroke: development and validation of new prognostic models. Stroke 2002; 33:1041-7.

17 Nabavi DG, Kloska SP, Nam EM et al. MOSAIC: multimodal stroke assessment using computed tomography: novel diagnostic approach for the prediction of infarction size and clinical outcome. Stroke 2002; 33:2819-26.

18 Counsell C, Dennis M. Systematic review of prognostic models in patients with acute stroke. Cerebrovasc Dis 2001; 12:159-70.

19 Weimar C, Konig IR, Kraywinkel K, Ziegler A, Diener HC; German Stroke Study C. Age and National Institutes of Health Stroke Scale Score within 6 hours after onset are accurate predictors of outcome after cerebral ischemia: development and external validation of prognostic models. Stroke 2004; 35:158-62.

20 Weimar C, Ziegler A, Konig IR, Diener HC. Predicting functional outcome and survival after acute ischemic stroke. J Neurol 2002; 249:888-95.

21 Muscari A, Puddu GM, Santoro N, Zoli M. A simple scoring system for outcome prediction of ischemic stroke. Acta Neurol Scand 2011; 124:334-42.

22 Smith EE, Shobha N, Dai D et al. Risk score for in-hospital ischemic stroke mortality derived and validated within the Get With the Guidelines-Stroke Program. Circulation 2010; 122:1496-504.

23 Michel P, Odier C, Rutgers M et al. The Acute STroke Registry and Analysis of Lausanne (ASTRAL): design and baseline analysis of an ischemic stroke registry including acute multimodal imaging. Stroke 2010; 41:2491-8.
24 Barber PA, Demchuk AM, Zhang J, Buchan AM. Validity and reliability of a quantitative computed tomography score in predicting outcome of hyperacute stroke before thrombolytic therapy. ASPECTS Study Group. Alberta Stroke Programme Early CT Score. Lancet 2000; 355:1670-4.

25 Blennow K, Wallin A, Uhlemann C, Gottfries CG. White-matter lesions on CT in Alzheimer patients: relation to clinical symptomatology and vascular factors. Acta Neurol Scand 1991; 83:187-93.

26 Wintermark M, Flanders AE, Velthuis B et al. Perfusion-CT assessment of infarct core and penumbra: receiver operating characteristic curve analysis in 130 patients suspected of acute hemispheric stroke. Stroke 2006; 37:979-85.

27 Wintermark M, Reichhart M, Thiran JP et al. Prognostic accuracy of cerebral blood flow measurement by perfusion computed tomography, at the time of emergency room admission, in acute stroke patients. Ann Neurol 2002; 51:417-32.

28 European Stroke Organisation Executive C, Committee ESOW. Guidelines for management of ischaemic stroke and transient ischaemic attack 2008. Cerebrovasc Dis 2008; 25:457-507.

29 DeLong ER, DeLong DM, Clarke-Pearson DL. Comparing the areas under two or more correlated receiver operating characteristic curves: a nonparametric approach. Biometrics 1988; 44:837-45.

30 Heldner MR, Zubler C, Mattle HP et al. National Institutes of Health stroke scale score and vessel occlusion in 2152 patients with acute ischemic stroke. Stroke 2013; 44:1153-7.

31 Derex L, Nighoghossian N, Hermier M et al. Influence of pretreatment MRI parameters on clinical outcome, recanalization and infarct size in 49 stroke patients treated by intravenous tissue plasminogen activator. J Neurol Sci 2004; 225:3-9.

32 Furtado AD, Smith WS, Koroshetz W et al. Perfusion CT imaging follows clinical severity in left hemispheric strokes. Eur Neurol 2008; 60:244-52.

33 Nezu T, Koga M, Kimura K et al. Pretreatment ASPECTS on DWI predicts 3-month outcome following rt-PA: SAMURAI rt-PA Registry. Neurology 2010; 75:555-61.

34 Dani KA, Thomas RG, Chappell FM et al. Computed tomography and magnetic resonance perfusion imaging in ischemic stroke: definitions and thresholds. Ann Neurol 2011; 70:384-401.

35 Puetz V, Khomenko A, Hill MD et al. Extent of hypoattenuation on $\mathrm{CT}$ angiography source images in basilar artery occlusion: prognostic value in the Basilar Artery International Cooperation Study. Stroke 2011; 42:3454-9.

36 Miteff F, Levi CR, Bateman GA, Spratt N, McElduff P, Parsons MW. The independent predictive utility of computed tomography angiographic collateral status in acute ischaemic stroke. Brain 2009; 132(Pt 8):2231-8.

37 Gonzalez RG, Lev MH, Goldmacher GV et al. Improved outcome prediction using CT angiography in addition to standard ischemic stroke assessment: results from the STOPStroke study. PLoS ONE 2012; 7:e30352.

38 Zhu G, Michel P, Aghaebrahim A et al. Prediction of recanalization trumps prediction of tissue fate: the penumbra: a dual-edged sword. Stroke 2013; 44:1014-9.

39 Lansberg MG, Straka M, Kemp S et al. MRI profile and response to endovascular reperfusion after stroke (DEFUSE 2): a prospective cohort study. Lancet Neurol 2012; 11:860-7.

40 Nagakane Y, Christensen S, Brekenfeld C et al. EPITHET: positive result after reanalysis using baseline diffusion-weighted imaging/ perfusion-weighted imaging co-registration. Stroke 2011; 42:59-64.

41 An H, Ford AL, Vo K et al. Early changes of tissue perfusion after tissue plasminogen activator in hyperacute ischemic stroke. Stroke 2011; 42:65-72.

42 Maas MB, Lev MH, Ay H et al. Collateral vessels on CT angiography predict outcome in acute ischemic stroke. Stroke 2009; 40:3001-5. 